\title{
Prevalence of Pathogens in Cystic Fibrosis Patients in Bahia, Brazil
}

\author{
Maria Angélica Santana, Eliana Matos, \\ Maria do Socorro Fontoura, Rosana Franco, \\ Danyella Barreto and Antônio Carlos M. Lemos
}

\author{
Octávio Mangabeira Hospital, Centre of Cystic \\ Fibrosis Reference of Bahia, Salvador, BA, Brazil
}

\begin{abstract}
Introduction. Recurrent respiratory infections account for most of the morbidity and mortality of cystic fibrosis patients. Materials and Methods. The objective was to determine the prevalence of pathogens isolated from lower respiratory tract secretions in cystic fibrosis patients. In this descriptive observational study, data from 69 patients was collected from medical records. Results. The microorganisms that were identified included $36.2 \%$ P. aeruginosa, $28.9 \% \mathrm{~S}$. Aureus, $4.3 \%$ K. pneumoniae, $1.5 \% \mathrm{H}$. influenzae, $1.5 \% \mathrm{E}$. coli, $1.5 \%$ S. maltoophilia, and in $27.5 \%$ the flora was normal. The prevalence of $P$. aeruginosa was $83 \%$ in patients under two years of age, demonstrating early colonization. Conclusion. P. aeruginosa and $S$. aureus were the most prevalent pathogens; there was also early infection/colonization by $P$. aeruginosa. This information will contribute to improved therapeutic measures for patients of the Bahia Cystic Fibrosis Reference Center.
\end{abstract}

Key Words: Cystic fibrosis, pathogens, Bahia, Brazil.
High morbidity and mortality rates incystic fibrosis patients are secondary to recurrent respiratory infections, which, when associated with this obstructive lung disease, lead to respiratory insufficiency, the main cause of death in these patients [1]. When cystic fibrosis was first described, most of the deaths occurred before five years of age as a consequence of Staphyloccocus infection of the lungs [2]. During the last three decades, $P$. aeruginosa has become the main pathogen in deathrelated lung infections, with the aggravating character of causing deterioration in lung function more rapidly than other infectious agents [2-4]. One of the reasons for this deterioration of lung function is the production of a specific Received on 16 November 2001; revised 18 August 2002. Address for correspondence: Dr. Maria Angélica Santana. Rua do Escravo Miguel, 134, Apto 101 - Ondina, 40170-000 Salvador, Bahia - Brazil. Phone: 5571 332-2356/Fax: 5571 2451905. E-mail: angelicasantana@terra.com.br

This study was conducted at the "Centro de Referência de Fibrose Cística da Bahia" (Bahia Reference Center for Cystic Fibrosis). Hospital Octávio Mangabeira, Bahia State Health Department. SPONSOR: Núcleo de Pesquisa em Pneumologia (Pneumology Research Center) - NUPEP

The Brazilian Journal of Infectious Diseases 2003;7(1):69-72 (C) 2003 by The Brazilian Journal of Infectious Diseases and Contexto Publishing. All rights reserved. lipopolysaccharide by $P$. aeruginosa [5]. The prognosis of CF has improved considerably after appropriate therapeutic protocols were implemented and due to the centralization of treatment in reference services. Mean survival, which was less than one year in the 1940's, increased to 32 years in 2001 [6]. The microbiology of $\mathrm{CF}$ is complex; the most prevalent pathogens are $P$. aeruginosa and $S$. aureus [2]. One factor that explains why patients suffering from $\mathrm{CF}$ have recurrent respiratory infections is the changes that are provoked in mucociliary transport in the respiratory tract [7]. Colonization of the respiratory tract in a patient with $\mathrm{CF}$ frequently follows by a sequence initiated with $S$. aureus or $H$. influenza, and then succeeded by $P$. aeruginosa $[3,8]$. Nevertheless, $P$. aeruginos a can colonize or infect during childhood, even during the first months of life, initially with the non mucoid form [9], and later on it changes to the mucoid phenotype [10], which is difficult toerradicate $[4,8]$. A vicious cycle of infection and inflammation is created and it is punctuated by acute exacerbations.

One of the limitations for microbiological studies of $\mathrm{CD}$ patients is that they experience periods of clinical stability with little or no secretion, and they can also have mild respiratory disease. However, a number of detailed epidemiological studies (incidence, prevalence, 
Table 1. General characteristics of patients $(\mathrm{N}=69)$

\begin{tabular}{lc}
\hline Age (years) & \\
Mean $( \pm$ SD) & $10.8 \pm 13.5$ \\
Median & 5 \\
Amplitude & $0.1-59$ \\
$\%<-16$ & $75 \%$ \\
Gender & \\
$\quad$ Male & $53.7 \%$ \\
Ethnic Group & \\
$\quad$ White & $33.9 \%$ \\
Mulatto & $62.0 \%$ \\
Black & $3.3 \%$ \\
\hline
\end{tabular}

Table 2. Prevalence of pathogens identified in cultures of secretions from the lower airways of patients who suffered from Cystic Fibrosis (N=69)

\begin{tabular}{lc}
\hline Pathogen & N $=\%$ of total \\
\hline P. aeruginosa & 36.2 \\
S. aureus & 27.4 \\
H. influenzae & 1.5 \\
K. pneumoniae & 1.5 \\
E. coli & 1.5 \\
Saprophytic flora & 27.5 \\
S. maltophilia & 1.5 \\
S. pneumoniae & 1.5 \\
S. aureus + Klebsiela & 1.5 \\
\hline
\end{tabular}

etc.) have been performed at different reference centers with the objective of improving the management of these infections [1]. The identification and knowledge of the behavior of these pathogens in cystic fibrosis patients in our community is relevant to the planning and development of therapeutic strategies.

\section{Objectives}

Describe the prevalence of pathogens found in lower airways of patients at the Bahia Reference Center for Cystic Fibrosis and determine how this prevalence varies in different patient age groups.

\section{Materials and Methods}

The variables of interest were gender, race, age and pathogens isolated from secretions of the lower airways. This was an observational descriptive study in which data were collected from medical records of patients between 1991 and 2001. The secretion samples from lower airways of 69 patients ( $68 \%$ of the patients cared for at the Reference Center) were obtained; spontaneous sputum accounted for $95 \%$, orotracheal suction, $2.2 \%$ and bronchoscopy, $2.2 \%$. In the distribution of patients according to age range, we considered the patient's age when the first sputum culture was made. 
Table 3. Prevalence of pathogens identified in secretions collected from the lower airways in patients who had Cystic Fibrosis, classified according to age group $(n=69)$

\begin{tabular}{ccccccccccc}
\hline $\begin{array}{c}\text { Age } \\
\text { (Years) }\end{array}$ & $\begin{array}{c}\text { \% of patients } \\
\text { with cultures }\end{array}$ & NF & PA & SA & KP & SA + PA & HI & SP & EC & SM \\
\hline $0<2$ & $27.3 \%$ & - & $83.3 \%$ & $16.6 \%$ & - & - & - & - & - & - \\
$2<6$ & $57.7 \%$ & $23.3 \%$ & $20.0 \%$ & $53.3 \% *$ & $6.7 \%$ & $6.7 \%$ & - & - & - & - \\
$6<11$ & $81.2 \%$ & $38.4 \%$ & $23.0 \%$ & $23.0 \%$ & $7.7 \%$ & - & - & $7.7 \%$ & - & - \\
$11<16$ & $100.0 \%$ & $22.2 \%$ & $33.3 \%$ & $22.2 \%$ & - & $11.1 \%$ & - & $11.1 \%$ & - & - \\
$16<25$ & $100.0 \%$ & $10.0 \%$ & $30.0 \%$ & $40.0 \% *$ & $10.0 \%$ & $9.9 \%$ & $10.0 \%$ & - & $10.0 \%$ & - \\
$25<35$ & $80.0 \%$ & $30.0 \%$ & $50.0 \% *$ & - & - & - & - & - & - & $10.0 \% * *$ \\
$>35$ & $88.8 \%$ & $33.3 \%$ & $55.5 \%$ & $22.2 \%$ & - & $28.8 \% *$ & - & - & - & - \\
\hline
\end{tabular}

* Associated with $K$. pneumoniae and $S$. aureus.

** Associated with $S$. maltophilia and P. aeruginosa.

$\mathrm{NF}=$ normal flora $; \mathrm{PA}=P$. aeruginosa $; \mathrm{SA}=S$. aureus $; \mathrm{KP}=K$. pneumoniae $; \mathrm{SA}+\mathrm{PA}=S$. aureus + P. aeruginosa $; \mathrm{HI}=H$. influenzae $; \mathrm{SP}=S$. pneumoniae $; \mathrm{EC}=E$. coli $; \mathrm{SM}=S$. maltophilia .

\section{Results}

The mean age was $10.8 \pm 13.5$ years (median 5 and amplitude of $0.1-59$ years); $75 \%$ of the patients were diagnosed during childhood ( $\leq 16$ years of age) and $25 \%$ as adults (>16 years of age); $53.7 \%$ were male; $33.9 \%$ were white, $62 \%$ mulatto and $3.3 \%$ were black. Table 1 shows the general characteristics of the population and Table 2 shows the prevalence of pathogens identified in the first culture of secretions from lower airways.

\section{Discussion}

As there have been no studies of the prevalence of pathogens in the lower airways of patients who suffer from Cystic Fibrosis in Brazil, this pioneer study is an important instrument to help determine the prevalence of pathogens in these types of patients. Data from the National Cystic Fibrosis Registry in 1996, which included 17,620 cystic fibrosis patients showed that $59.9 \%$ were positive for $P$. aeruginosa, $37.5 \%$ for $S$. aureus, $15.4 \%$ for $H$. influenzae, $3.6 \%$ for B. cepace a and $3.9 \%$ for S. maltophilia. 1 In our study, we found similar pathogens, but with lower prevalences than those observed in the above study (Table 1); this could be due to differences in the size and origin of the samples. When prevalence was evaluated by age group, we observed that $P$. aeruginosa increased with age, but with a high prevalence of around $20 \%$ during the first years. In our study, we found a high frequency (83\%) of $P$. aeruginosa in children younger than two years, which is considerably higher than the prevalence found in the study mentioned above. This is worrisome since $P$. aeruginosa is associated with accelerated deterioration of lung function. Many factors contribute to the early colonization of airways by $P$. aeruginosa in $\mathrm{CF}$ patients. In our series, we observed that patients who were less than two years old, and who had an infection caused by $P$. aeruginosa, had a history of prolonged hospitalization, including stays in intensive care units, and previous use of antibiotics. This fact might be responsible for the differences observed between our study and the national study indicated above. Moreover, an important limitation in our study is the small sample size, which became more evident when we evaluated the prevalence in different age groups.

\section{Conclusion}

We found a high prevalence of $P$. aeruginosa in cultures collected from the lower airways of patients who suffered from $\mathrm{CF}$; this occurred even during the first two years of age, which is evidence of early colonization. This information will help with adequate 
therapeutic planning and with the development of preventive strategies at the Bahia Reference Center for Cystic Fibrosis.

\section{Refrences}

1. Fitz-Simmons S.C. The changing epidemiology of cystic fibrosis. J Pediatr 1993; 1:122.

2. Hoiby N. Microbiology of cystic fibrosis. In: Hodson M., Geddes D., eds. Cystic Fibrosis 1995:75-98.

3. Doggett R.G. Incidence of mucoid Pseudomonas aeruginosa from clinical sources. Applied Microbiol 1969; 18:936-7.

4. LiPuma J. Cystic Fibrosis. Clin Chest Med 1998;19:473-86.

5. Ernst R.K., et al. Specific lipopolysaccharide found in cystic fibrosis airway Pseudomonas aeruginosa. Science 1999;286:1561.

6. Beverly P.W. Cystic Fibrosis: 1997. Radiology 1997;204:1-10.

7. Pier G.B., et al. Role mutant CFTR in hypersusceptibility of cystic fibrosis patients to lung infection. Science 1996; $271: 64$.

8. Pedersen S.S. Lung infection with alginate - producing mucoid Pseudomonas aeruginosa in cystic fibrosis. APMIS 1992;100(suppl.28):5-79.

9. Kosorok M.R., et al. Comprehensive analysis of risk factors for acquisition of Pseudomonas aeruginosa in young children with cystic fibrosis. Pediatr Pulmonol 1998; $26: 81$.

10. Renders N.H., et al. Exchange of Pseudomonas aeruginosa strains among cystic fibrosis siblings. Res Microbiology 1997; 148:447.

11. David M. Orenstein, Glenna B. Winnie and Harold Altman. Cystic fibrosis: A 2002 update. The Journal of Pediatrics. February 2002. 